\title{
Über Veränderungen der abnorm gekrümmten Schwanzwirbelsäule des Kaninchens.
}

\author{
Von
}

\author{
Prof. Dr. Ribbert \\ in Zürich.
}

Mit Tafel XXXI.

Eingegangen am 14. Februar 1898.

Bei meinen experimentellen Untersuchungen über die Transplantation verschiedener Gewebe $\left.{ }^{1}\right)$ hatte ich u. A. auch Knochenstiickchen benutzt, die ich aus der Schwanzwirbelsäule des Kaninchens entfernte. Nun brachte ich aber nicht nur völlig abgetrennte Gewebstheile in Anwendung, sondern wollte auch prüfen, wie sich diejenigen verhielten, die zunächst ohne Unterbrechung der Ernährung an eine benachbarte Stelle transplantirt wurden, um erst, nachdem sie dort angewachsen waren, ans dem ursprünglichen Zusammenhange ganz gelöst zu werden. So führte ich $\mathfrak{u}$. A. die von Haut befreite Schwanzspitze des Kaninchens in eine Hauttasche des Riuckens nahe der Schwanzwurzel und ließ sie dort, indem ich sie durch einige Nähte fixirte, anheilen. Dabei musste ich nun das Organ über seine dorsale Fläche so biegen, dass es ungefähr Ringform annahm. Durch diesen Umstand aber kam ich auf den Gedanken, die übernormale Krümmung zu benutzen, um entsprechend einem schon länger gehegten Wunsche festzustellen, auf welche Weise Knochen bei geänderter Inanspruchnahme ihre Gestaltung ändern, wie also in unserem speciellen Falle die Wirbel sich der Biegung anpassen wïrden. Solche Untersuchungen erschienen ja bei dem großen Interesse, welches den funktionellen Anpassungen (Roux) des Skeletsystems entgegengebracht und besonders durch die bekannte Arbeit von J. WOLFF genährt wurde, um so mehr angebracht, als sie bisher 
fast ausschließlich am fertigen anatomischen Präparat und nur in ganz bescheidenem Umfange auf experimentellem Wege vorgenommen wurden. Daher sind wir zwar gut unterrichtet uber Form und Bau derjenigen Knochen, auf welche längere Zeit geänderte funktionelle Bedingungen eingewirkt haben, dagegen wissen wir nur sehr wenig. darüber, wie denu diese Endresultate zu Stande gekommen sind. In dieser Richtung hoffte ich weitere Aufklärung zu gewinnen.

Da die zahlreichen hierhergehörigen anatomischen Untersuchungen und die Gesichtspunkte, anf die es ankommt, als bekannt vorausgesetzt werden dürfen, so will ich ohne weitere Einleitung an die Schilderung meiner Experimente herangehen, die ich an 16 Kaninchen anstellte.

Jene übernormale Krümmung der Schwanzwirbelsäule behielt gewöhnlich nicht dauernd die anfänglich ziemlich regelmäßige Rundung. Meist bogen sich zwei etwa in der Mitte gelegene Wirbel stärker gegen einander, so dass hier eine deutliche Knickung entstand. Das war besonders dann ausgesprochen, wenn ich die abgehäutete Spitze statt unter die Rückenhaut, in den basalen Abschnitt des Schwanzes selbst einführte, also die Intensität der Biegung verstärkte, den Ring enger machte. Da mir nun jene Knickung für meine Zwecke sehr geeignet schien, so suchte ich sie mehrfach dadurch zu befördern, dass ich den gekrimmten Schwanz mit Heftpflasterstreifen etwas zusammendriickte, so dass seine Form eine flachovale wurde. Auch habe ich versucht, ohne die Spitze subentan zu befestigen, lediglich mit Heftpflaster zum Ziele zu kommen, kanu dies Verfahren aber nicht empfehlen, weil die Thiere die Streifen gewöhnlich bald abrissen. Am besten verfährt man also so, dass man den Schwanz zunächst in der gekrümmten Stellung anwachsen lässt, um dann erst, wenn nöthig, das Heftpflaster in Anwendung zu bringen. Reißen dann die Thiere den Verband $\mathbf{a b}$, so kann er leicht, ohne wesentliche Änderung der ursprünglichen Biegung wieder angelegt werden.

Die Untersuchung der gekrümmten Schwänze wurde in rerschiedenen Intervallen von 6-105 Tagen rorgenommen.

Die amputirten Stücke wurden in ZeNKER'scher Lösung gebärtet und dann entkalkt.. Die Schnitte wurden natürlich in sagittaler Richtung und meist so angelegt, dass sie drei Intervertebralscheiben, also zwei ganze Wirbelkörper und Theile der angrenzenden umfassten und dass die mittlere Scheibe der stärksten Biegung bezw. ،der Kuickung eutsprach. 


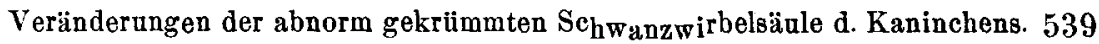

Je längere Zeit verflossen war, desto weniger ließen sich die gebogenen Organe gerade strecken, wie es bei den normalen der Fall ist, man hätte sonst eine Zerreißung an der konkaven Seite herbeifübren müssen. Es hatte also eine Fixation in der neuen Stellung stattgefunden, die z. B. 3 Wochen nach Beginn des Versuchs schon sehr ausgesprochen sein konnte.

Allerdings hängt dieses, wie das ganze Versuchsresultat von der Intensität der Krümmung ab. Wenn diese eine gleichmäßig ringförmige bleibt, pflegt sich entweder keine wesentliche Änderung oder nur eine geringe einzustellen. Nur bei deutlich winkeliger Knickung sind die Ergebnisse befriedigend. Doch sollte sie auch nicht über cin gewisses Maß hinaus getrieben werden, da einerseits, wie wir sehen werden, die Knochen an der Konvexität in der Nähe der Zwischenwirbelscheiben einreißen können und andererseits auch die zu stark gespannte Haut nekrotisch wird. Derartige Experimente sind zwar auch nicht werthlos, immerhin aber nicht ganz in gleichem Umfange verwerthbar, wie die nach jeder Richtung gut gelungenen.

Außer von der Stärke der Krümmung hängt das Resultat auch rom Alter der Thiere ab. Junge reagiren leichter als ältere.

Aber auch bei Beachtung dieser Cautelen habe ich nicht in allen Experimenten vollen Erfolg gehabt. In einzelnen blieben die Veränderungen außerordentlich geringfiugig, ohne dass es mir klar geworden wäre, worin ich den Grund zu suchen hatte. Doch war in keinem dieser Versuche die Biegung bis zur winkeligen Knickung ausgedehnt worden. Immerhin hatte ich an anderen Objekten, die auch nicht stärker gekriummt waren, gute Resultate.

In allen befriedigender verlaufenden Fällen aber waren die sich einstellenden Veränderungen durchaus charakteristisch und in der Hauptsache ïbereinstimmend. Im Einzelnen freilich variiren sie etwas. Das hing natiurlich einerseits von der Versuchsdauer ab, die aber doch wiederum nicht so maßgebend war, dass nun alle gleich alten Präparate gleich ausgesehen hätten. Vielmehr verlief der Process bei dem einen Thier rascher als bei dem anderen, so dass Objekte, die der Zeit nach erheblich verschieden waren, dieselben Verhältnisse darbieten konnten. Andererseits variirten die Ergebnisse je nach der Intensität der Krümmung.

Die Reichhaltigkeit der Resultate wurde nun noch beträchtlich dadurch gesteigert, dass in den einzelnen Objekten gewöhnlich nicht nur an einer Stelle die Folgezustände sich geltend machten, sondern dass außer an der am stärksten gebogenen Synchondrose auch an 
den beiden benachbarten deutliche Veränderungen hervortraten. Da diese nun nicht überall gleich intensiv und gleich vorgeschritten waren, so wurden bei demselben Thiere mehrere verschiedene Stadien gewonnen.

Die Beschreibung der Präparate soll nicht mit dem jüngsten beginnen. Es ist übersichtlicher, wenn ich zuerst ein charakteristisch verändertes Objekt schildere, um daran frühere und spätere Stadien und die ersten Anfänge anzuschließen.

Es wird sich aber vor Darstellung der Versuchsergebnisse empfehlen, auf die normalen Befunde am Kaninchenschwanze in Kürze einzugehen. Ich thue das an der Hand der halbschematischen Fig. 1.

Die Wirbelkörper des Kaninchenschwanzes baben im Wesentlichen die Form und den Bau von Fingerphalangen. In der Figur sind die Enden zweier Körper $a$ und $b$ gezeichnet, ihre ganze Länge würde das Vier- bis Fünffache betragen. Sie sind leicht gegen einander geneigt entsprechend der physiologischen dorsalen Krümmung des Schwanzes. Die konvexe Seite der Figur ist also die ventrale des Organs. Auf die Diaphyse des Wirbelkörpers folgt zunächst je eine epiphysäre Knorpelscheibe $E$, darauf in dïnner Lage die knöcherne Epiphyse, die gegen die konvexe Seite hin etwas mächtiger entwickelt ist als gegen die konkave. Nun kommt die Zwischenwirbelscheibe $J$, die eine leicht keilförmige Gestalt hat, $d$. h. an der Konvexität etwas breiter ist als an der Konkavität. Das tritt meist noch etwas ausgesprochener hervor, als es in der Figur der Fall ist. Der Intervertebralknorpel schließt den Chordakern $G$ ein, der bei jüngeren Thieren stets vorhanden ist, im sagittalen Schnitt meist eine länglich ovale etwas unregelmäßige Form hat und sich gegen die Konkavität der Krümmung gewöhnlich etwas verjüngt. Zuweilen ist die Gestalt des Kerns mehr rundlich. Bei alten Thieren fehlt der Knorpel der Epiphysenlinie und der epiphysäre Knochen ist von der Diaphyse nicht mehr getrennt. Auch der Chordakern kann dann völlig fehlen. $\mathrm{Zu}$ meinen Versuchen dienten meist juingere Thiere.

$\mathrm{Zu}$ beachten ist endlich noch, dass trotz der leichten gegenseitigen Biegung die Längsachse der Wirbelkörper wegen der keilförmigen Gestalt der Intervertebralscheibe gewöhnlich nahezu senkrecht auf dieser steht. Doch kommt es auch zuweilen vor, dass sie etwas geneigt erscheint und mit der Epiphysenlinie einen nach oben stumpfen, nach unten spitzen Winkel bildet. 
Diese normalen Verhältnisse finden sich nun, wie Fig. 2 zeigt, an einem ersten Objekt 62 Tage nach Beginn des Versuchs in folgender Weise abgeändert. Zunächst ist in Folge der Biegung die Intervertebralscheibe $J$ außen breiter, innen schmaler geworden. Das beruht vor Allem auf einer Dehnung an der Konvexität, weniger auch, da die Krümmung nur eine mäßige war, auf einer Kompression an der Konkavität. Zugleich ist der Chordakern $G$ verschoben und liegt mehr als vorher in der ventralen Hälfte der Synchondrose. Seine Gestalt ist ebenfalls verändert. Er ist in senkrechter Richtung abgeplattet und von unten her eingedrückt. Im Zusammenhang damit ist die streifige, dem schichtweisen Aufbau entsprechende Zeichnung der Zwischenwirbelscheibe eine andere geworden. Sie folgt der Biegung des Chordakerns. An der Konvexität zieht sich die Scheibe geradlinig rom Rande des einen Knochens zum anderen heruber, während sie in Fig. 1 etwas eingesunken erschien. Sie ist also durch die Biegung gegen die Konvexität gedrängt, weil die Knochen an der Konkavität einander genähert wurden.

Die epiphysären Knochen bilden an der Innenseite der Biegung eine wesentlich diunnere Lage als außen. Es ist indessen fraglich, ob darin eine Folge der Kompression zu sehen ist, da ja auch in der Norm derselbe, wenn auch meist geringere Unterschied hervortritt. Die Epiphysenknorpel sind überall gleich breit.

Alle diese Abweichungen sind die nächsten mechanischen Folgen des Eingriffes. An sie haben sich nun vor Allem wichtige Veränderungen an der Innenseite des Biegungswinkels angeschlossen. Wir bemerken hier eine beträchtliche Neubildung von Knorpel im Zusammenhang mit der Synchondrose und von Knochen an der angrenzenden Unterfläche beider Diaphysen. Die Intervertebralscheibe setzt sich direkt in den hyalinen Knorpel $K$ fort, hat also eine bedeutende Größenzunahme erfahren. Ihre friihere Grenze lag etwas unter der durch eine punktirte Linie bezeichneten Stelle. Der neue Knorpel bildet ein etwa rechteckiges Feld, welches nach oben hin einen kegelförmigen Ausläufer in die Synchondrose hineinschiebt und mit beiden Epiphysenknorpeln zusammenhängt, nach unten sich von dem anstoßenden Bindegewebe in einer nicht sehr scharfen, aber doch erkennbaren Grenze absetzt. Rechts and links schließen sich nun an den Knorpel Bezirke neuen Knochergewebes, $K n a$ und $K n b$, an. Bei $K n b$ ist das am deutlichsten. Der Wirbelkörper hat einen aus dichter Masse bestehenden kegelförmigen Vorsprung bekommen, dessen eine Seite von jenem Knorpel, 
dessen andere von Periost begrenzt wird. Bei $K n a$ ist diese Knochenneubildung, zumal in dem der Figur zu Grunde liegenden Schnitt, weit geringfügiger.

Die Grenze des neugebildeten Knorpels und Knochens verläuft nicht in der Richtung der Epiphysenlinie, vielmehr bildet sie mit ihr an dem fritheren Rande der Zwischenwirbelscheibe einen stumpfen Winkel.

An der Konvexität der Biegung zeigen die Wirbelkörper in diesem Präparat nur geringe Veränderungen. Am meisten treten sie noch am Wirbel $a$ hervor. Seine Begrenzung verläuft bei $z$ nicht mehr in gerader Linie gegen das Ende des Epiphysenknorpels. Sie ist vielmehr in der Nähe des letzteren etwas ausgezackt und reducirt. Denn die von der Mitte der Diaphyse aus fortgesetzt gedachte Randlinie würde etwas weiter außen verlaufen, als es hier der Fall ist. Der Wirbelkörper $b$ zeigt nur Andentungen des gleichen Verhaltens.

Die Längsachse der Diaphysen steht, besonders die von $a$, schräg zur Epiphysenlinie, bildet mit dieser einen nach unten offenen spitzen Winkel.

An die primären rein mechanischen Abweichungen der Synchondrose haben sich also sehr charakteristische Processe angeschlossen, nämlich erstens und vor Allem Neubildung von Knorpel und Knochen an der Konkavität. Ersterer entwickelt sich, wie aus den folgenden Versuchen noch klarer hervorgehen wird, aus der Zwischenwirbelscheibe, ist aber in seiner Struktur wesentlich von ihr verschieden. Denn er hat alle Eigenschaften des hyalinen Knorpels, verhält sich also so wie die Intervertebralscheibe in ihrer ersten embryonalen Anlage. Darin ist ein neues Beispiel für die Erscheinung gegeben, dass bei der Regeneration das neugebildete Gewebe zunächst einem friheren entwickelungsgeschichtlichen, weniger differenzirten $\mathrm{Zu}$ stand des zu ersetzenden entspricht. (Vgl. dariiber den eingangs citirten Aufsatz.)

Die Knorpelneubildung aus der Zwischenwirbelscheibe ist in so fern eine bemerkenswerthe Thatsache, als der übrige Knorpel unseres Körpers zu denjenigen Geweben gehört, die außerordentlich wenig. Neigung zur Proliferation zeigen. Hier dagegen sehen wir ein beträchtliches Wachsthum. Es ist mir das auch schon friiher aufgefallen, als ich meine Versuche über die experimentelle Erzeugung eines Chordoms aus dem Gallertkern der Zwischenwirbelscheibe anstellte ${ }^{1}$.

1) Verhandl. d. Kongresses f. innere Med. 1595. München. 
Anch in diesen Experimenten sah ich aus der Synchondrose hyalinen Knorpel herauswachsen, dort wo ich mit dem Messer eingestoßen hatte, um den Chordakern hervorquellen zu lassen.

Jene Knochenneubildung erfolgt nun zum großen Theil unter Vermittelung des neuen Knorpels nach Art des normalen epiphysären Processes. Dementsprechend sieht man bei starker Vergrößerung die bekannte, wenn auch nicht sehr ausgeprägte Gruppen- und Reihenstellung der Knorpelzellen senkrecht zum Knochen. Doch ist, wie sich später deutlicher ergeben wird, das Periost an den Wachsthumsvorgängen nicht unbetheiligt. Die neuen Knochenbälkchen sind mit Osteoblasten reichlich besetzt.

Neben den Neubildungsprocessen finden sich an der Konvexität regressive Veränderungen in Gestalt einer Einschmelzung vou Knochensubstanz. Am Wirbelkörper $a$ ist bei $z$ subperiostal ein Schwund von Knochen vor sich gegangen und seine Grenze ist unregelmäßig. Bei stärkeren Linsen lässt sich leicht feststellen, dass die Anfsaugung unter Mithilfe von Riesenzellen geschieht, die in einem zellreichen Periostgewebe liegen. Die unter dem in Resorption begriffenen Abschnitt gelegenen Kuochenbälkchen sind mit regelmäßigen Lagen von Osteoblasten versehen. Unter der Einschmelzung: also erfolgt Neubildung.

Wir gehen jetzt zu einem Versuch von 42 tägiger Dauer über. Ihm entspricht die Fig. 3. Die Biegung war etwas geringer, aber nichtsdestoweniger sind die Veränderungen sehr ausgeprägt. Wir sehen an der Konkavität den neuen Knorpel, $K$, noch reichlicher entwickelt als in Fig. 2. Fr bildet ein groles rechteckiges Feld, an welches beiderseits der junge Knochen $K n a$ und $K n b$ anstößt, dessen Form auch hier die eines Kegels ist. Sein Gefüge ist weniger dicht, die Weite seiner Markräume beträchtlicher als in dem vorigen Falle. Ein weiterer Unterschied besteht darin, dass hier die kegelförmigen Verdickungen an beiden Diaphysen in gleicher Ausbildung vorhanden sind.

Die Grenze des neuen Knorpels gegen die alte Intervertebralscheibe verläuft ziemlich geradlinig und ist sehr deutlich sichtbar. Dadurch kann man den Umfang der neu entstandenen Theile klar bestimmen. Denn was unterhalb jener Grenze liegt, ist neugebildet. Die Beurtheilung ist aber durch den Umstand noch wesentlich erleichtert, dass der Knorpel der Epiphysen $E$ nicht mit dem aus der Zwischenwirbelscheibe herausgewachsenen zusammenhängt, von ihm vielmehr durch eine Schicht faseriger Substanz getrennt ist. Gerade 
aus diesem Befunde aber lässt sich andererseits entnehmen, dass an der Bildung des Knorpels $K$ lediglich die Intervertebralscheibe betheiligt ist.

Die unteren Grenzen der Epiphysenknorpel stoßen in einer bei $b$ horizontal verlaufenden, bei $a$ schräg abfallenden Linie an den neuentstandenen Knochen an, der auch hier theils chondrogen, theils periostal entstanden ist und dessen Bälkchen Osteoblastenbeläge aufweisen.

Eine bemerkenswerthe Differenz gegenüber dem vorigen Präparat ist dadurch gegeben, dass der alte Knochen, ans dem die Vorsprïnge Kn $a$ und $K n b$ herausgewachsen sind, nicht mehr so gut erhalten ist, wie es dort der Fall war und wie es hier noch an der Konvexität hervortritt. Er bildet nicht mehr eine gleichmäßig breite Schicht, sondern ist unterbrochen und spongiös gebaut. Das deutet auf partielle Resorptionsprocesse hin, die indessen in dem feineren histologischen Verhalten keine Begründung finden. Von Osteoklasten ist nichts wahrzunehmen. Ein etwa vorher vorhandener Einschmelzungsvorgang muss also bereits abgelaufen sein. Da aber die Abweichung im Bau vorhanden ist und die Begrenzung der Diaphyse nicht mehr so klar hervortritt, so ist es etwas schwieriger, das Verhalten der Längsachse der Wirbelkörper zu der Epiphysenlinie zu bestimmen. Immerhin kann man sehen, dass auch hier die letztere in a etwas schief zu jener Achse steht, so dass sie mit ihr einen nach unten offenen spitzen Winkel bildet. Bei $b$ tritt das kaum dentlich hervor. Hier erscheint aber der Epiphysenknorpel etwas konvex gegen die $Z$ wischenwirbelscheibe gebogen, die nun ihrerseits noch an der Konvexität eine charakteristische, in Fig. 2 fehlende Veränderung aufweist. Wir sehen sie hier nämlich theilweise in einen hyalinen Knorpel, $K_{1}$, umgewandelt, der sich nach oben leicht vorwölbt. Dass es sich nicht etwa um dieselbe Erscheinung wie an der konkaven Seite handelt, geht aus den Lagebeziehungen ohne Weiteres hervor. Der Knorpel $K_{\mathrm{t}}$ ist eben an die Stelle eines Theils der Intervertebralscheibe getreten, hat sich dabei allerdings so vermehrt, dass er nach oben etwas prominirt. Er hängt beiderseits mit dem Epiphysenknorpel zusammen. Seine Entstehungsbedingungen sind denn auch, wie wir weiter unten sehen werden, ganz andere.

Etwas Neues wird ferner noch dargestellt durch einen relativ umfangreichen Knochenkern, $K n$, der im Bereich der Zwischenwirbelscheibe entstanden ist, an drei Seiten von Knorpel und nur nach unten von der faserigen Substanz begrenzt wird. 
Die folgenden Präparate habe ich in den Fig. 4, 5 und 6 in geringerer, der Fig. 1 entsprechenden Vergrößerung dargestellt.

Die Fig. 4 giebt uns ein Stadium ron 24 Tagen wieder. Die winkelige Knickung ist beträchtlich, die Folgezustände sind sehr ausgesprochen. Der an der Konkavität entstandene Knorpel, $K$, rerbreitert sich nach abwärts erheblich und gewinnt so die Form eines Kegels. Er liegt der Unterfläche des Wirbelkörpers $b$ eine Strecke weit glatt an. Dann erst findet sich ein kleiner Knochenvorsprung $K n b$. Auf der anderen Seite verläuft die Knorpelgrenze weniger schräg, sie stößt aber nirgendwo an die alte Unterfläche des Wirbelkörpers, sondern uiberall an neugebildeten, kegelförmig vorragenden Knochen, Kn $a$, an, dessen Masse hier also weit größer ist als bei $b$.

Die Grenze des Knorpels $\boldsymbol{K}$ und der Zwischenwirbelscheibe $J$ ist ziemlich gut erkennbar, von den Epiphysenknorpeln ist jener durch je ein diunnes Knochenbälkchen getrennt, so dass sich also seine Entstehung anch hier lediglich auf die Intervertebralscheibe zuriickfuhhren lässt. Letztere zeigt die oben erwähnte Verjüngung nach unten sehr dentlich und da andererseits der neue Knorpel sich nach der entgegengesetzten Seite verbreitert, so bilden beide Felder zusammen eine sanduhrähnliche Figur.

Der Chordakern, $G$, ist auf einen quer verlaufenden Streifen zusammengepresst.

Die Epiphysenknorpel zeigen eine Veränderung, die in den vorigen Objekten fehlte. Sie verdünnen sich nämlich gegen die Konkavität der Krümmung hin etwa um die Hälfte. Wir dürfen annehmen, dass dies unter dem Einfluss der hier einwirkenden Kompression geschehen ist.

An der Konvexität ist die in Fig. 3 beschriebene Knorpelneubildung ausgeblieben. Dagegen findet sich ungefähr an der gleichen Stelle auch hier ein Knochenkern, $x$, der aber einen größeren Umfang hat als dort. Er ist einerseits durch den Epiphysenknorpel, andererseits durch die Intervertebralscheibe begrenzt und bängt nach nuten mit der Epiphyse, seitlich mit der Diaphyse zusammen. Diese zeigt am freien Rande in der Nähe des Knochenkerns eine zackige Begrenzung, in deren Vertiefungen einzelne Riesenzellen liegen. Auf der anderen Seite, bei $z$, ist der Knochenrand zwar nur wenig unregelmäßig gebaut, aber dentlich reducirt, etwa in gleichem Sinne wie in Fig. 2. Bei starker Vergrößerung finden sich auch hier vereinzelte Osteoklasten. 
Die Längsachse der beiden Wirbelkörper steht nahezu senkrecht auf der Epiphysenlinie, verhält sich also nngefähr so wie im normalen Präparat (Fig. 1).

Fig. 5 zeigt die nach geringer Biegung eingetretenen Veränderungen 42 Tage nach Beginn des Experiments. Die Zwischenwirbelscheibe, der Chordakern und die Epiphysenknorpel zeigen keine Abweichungen, die nach dem Vorhergehenden noch einer besonderen Besprechung bedürften.

An der Konkavität sehen wir ausgedehnte Knorpelneubildung. Sie erstreckt sich flïgelförmig beiderseits unter die Wirbelkörper, besonders weit unter $a$. Die geringe Knochenneubildung $K n b$ findet sich zum größten Theil zwischen Knorpel und Diaphyse, während sie auf der anderen Seite, $K n a$, umfangreicher ist und einen flachen Kegel bildet, der aus relativ dünnen Bälkchen besteht und zum großen Theil periostealer Abkunft ist. Die Osteoblastenbeläge sind sehr reichlich entwickelt.

Fig. 6 giebt ausgedehnte Umgestaltungen an einem alten Thiere nach 72 Tagen wieder. Epiphysenknorpel und Chordakern fehlen. An der Konkavität bemerkt man den neuen Knorpel $K$ und den gleichfalls neven Knochenkegel $K n b$. Der Wirbelkörper $a$ zeigt an seiner unteren Seite keine wesentlichen Veränderungen.

Die stärksten Abnormitäten treten an der Konvexität und zwar vor Allem an dem Wirbelkörper $b$ hervor. Er ist bei $z$ um ein großes etwa dreieckiges Stiick reducirt, iiber dessen Umfang man eine Vorstellung gewinnt, weun man den Diaphysenrand und die Epiphysenlinie nach oben verlängert bis sich beide treffen. Es ist also von oben her viel Knochen eingeschmolzen worden, aber nicht ohne dass sich eine neue dicke, wenn anch unregelmäßige Lage gebildet hätte, welche die Knochenmarkhöhle wieder abschließt. Durch die Resorption und durch die an der Konkavität erfolgende Nenbildung ist die Diaphyse in ihrer Form sehr verändert. Es sieht so aus, als sei sie in der Nähe ihres Endes abgeknickt worden. Der Wirbelkörper $a$ hat im Ganzen ungeführ die normale Gestalt. Doch ist er diinner und zumal seine obere Begrenzung ist durchbrochen und etwas uneben. Auch hier sind offenbar Einschmelzungen vor sich gegangen.

Mit diesen an der Hand von fünf Präparaten gewonnenen Schilderungen älterer Stadien der experimentellen Resultate wollen wir uns begnügen. Ich könnte noch eine Reihe anderer Objekte beschreiben, doch wiirde in der Hauptsache immer nur wieder Dasselbe 
zu sagen sein. Die Unterschiede beziehen sich im Wesentlichen nur auf Form und Intensität des Knorpel- und Knochenwachsthums. Principielle Verschiedenheiten treten nicht hervor.

Wie verhalten sich nun die friheren Stadien? Was lehren sie über die allmähliche Entstehung der besprochenen Verhältnisse?

An einem sechstägigen Objekt sieht man noch nichts Anderes als die mechanischen Folgeerscheinungen, also Kompression der Zwischenwirbelscheibe an der konkaven Seite, Dehnung an der konvexen. Der Chordakern ist in der geschilderten Weise nach oben gedrängt.

Die Zusammendrückung der Intervertebralscheibe beginnt nun aber nicht genau an ihrem unteren Rande. Vielmehr sind die näher zusammenstehenden Kanten der Diaphysen zugleich ein wenig nach oben gerückt, an der Seite jener Scheibe etwas gegen die Konvexität verschoben, während sie im normalen Zustande gewöhnlich (Fig. 1) etwa um ebenso viel iber die Synchondrose nach unten herausragen. Dadurch sind die tiefsten Lagen der letzteren außer dem Bereich der Kompression und springen leicht konvex in den Biegungswinkel vor. Dieser Umstand ist für das Verständnis der Wachsthumsvorgänge von Wichtigkeit.

Die Epiphysenknorpel zeigen im Bereich der stärksten Druckwirkung, d. h. dort, wo auch die Intervertebralscheibe gepresst ist. eine deutliche, wenn auch nicht hochgradige Dickenabnahme.

An einem achttägigen Präparat sind an der Innenseite der Krümmung die Verhältnisse in der Hauptsache die gleichen. Nur ragt die Intervertebralscheibe nach unten etwas mehr vor, ohne aber deutliche Neubildung erkennen zu lassen. An der Konvexität findet sich dagegen eine beachtenswerthe Veränderung. Hier ist nämlich die Zwischenwirbelscheibe von ihrer Oberfläche her anf der einen Seite parallel dem Epiphysenknorpel und dicht an ihm eingerissen, so dass eine unregelmäßig trichterförmige Öffnung entstanden ist, an deren Rand die obersten horizontal gestreiften Lagen der Synchondrose scharf abschneiden. Die Lücke ist durch ein zellreiches, vorwieg*end aus Spindelzellen bestehendes Gewebe ausgefüllt, welches viele den Rissrändern anliegende Riesenzellen enthält.

Ein fünfzehntägiges Objekt bietet folgende Befunde: Durch die in diesem Falle beträchtliche Biegung ist die Intervertebralscheibe in ihrem inneren Drittel stärker als sonst zusammengedrickt und färbt sich hier diffus dunkler. Ihre Kerne färben sich schlecht und stellenweise gar nicht. Die Vorwölbung ihrer untersten Lagen ist 
auch hier sichtbar. Die unter der Druckwirkung stehenden inneren Abschnitte der Epiphysenknorpel sind erheblich verdünnt und blass gefärbt, die Kerne treten vielfach gar nicht mehr hervor. An der Konvexität ist die Intervertebralscheibe sehr stark geradlinig gespannt, ohne eingerissen zu sein. Auch die anstoßenden Weichtheile sind in gleichem Sinne gedehnt und liegen der Scheibe und den angrenzenden Knochentheilen dicht an. In letzteren finden sich Resorptionserscheinungen. Der Knochen ist an seinem Rande rom Epiphysenknorpel an eine Strecke weit, ähnlich wie in Fig. 2 und 4 bei $x$ und $z$, uneben, arrodirt und mit einer Schicht zellreichen Gewebes bedeckt, zu welchem in Gruben liegende Riesenzellen gehören. An der anderen Diaphyse sind ähnliche Veränderungen, aber nur geringfügig vorhanden.

Ein zweites Präparat von fünfzehn Tagen lässt auch an der Konkavität schon mehr erkennen. Die Zwischenwirbelscheibe ragt hier ausgesprochener vor und zeigt bereits den Anfang einer Bildung hyalinen Knorpels, in Gestalt kleiner Inselchen, in denen die Zwischenwirbelsubstanz hyalin geworden und durch Hämalaun blau gefärbt ist. Die angrenzenden Unterflächen der Wirbelkörper bieten ebenfalls schon den Beginn einer Neubildung. Sie sind uneben, das Periost hat sich in eine zellreiche Lage umgewandelt und zur Entstehung flacher aus dem Knochen herauswachsender osteophytärer Bälkchen gefïhrt. Hier haben wir also den Beginn der in Fig. 2-6 deutlicher ausgeprägten Veränderungen. Nun ist aber ferner bemerkenswerth, dass nicht nur Knorpel und Knochen Neubildungsvorgänge erkennen lassen, dass vielmehr auch die tibrigen Weichtheile im Winkel der Krümmung analoge Erscheinungen darbieten, zu deren Verständnis noch einige topographische Verhältnisse erörtert werden müssen. Am normalen Schwanz grenzt an die Synchondrose und die Diaphysen zunächst eine dünne Lage längsgestreiften Bindegewebes an. Darauf folgt ein ziemlich dickes längsrerlaufendes Ligament und nun wieder Zellgewebe und darin quergestreifte Mnskulatur. In den gebogenen Organen ist nun das Längsband im Kriimmungswinkel von der Zwischenwirbelscheibe und den angrenzenden Knochenabschnitten abgeriickt, verläuft gerade und schließt so den Winkel zu einem dreieckigen Raum ab, der leer sein würde, wenn nicht die hier befindlichen Gewebe, Periost, Perichondrium und Bindesubstanz sich vermehrt und ihn ausgefüllt hätteu. Demgemäß finden wir hier ein Gewebe, welches sich rorwiegend aus spindeligen, protoplasmareichen Zellen aufbaut, und sich nur undeutlich in die 
chondralen, periostalen und bindegewebigen Abschnitte sondert. Es geht in die Synchondrose ohne scharfe Grenze über und setzt sich ebenso direkt fort in die zellreiche knochenbildende Periostschicht.

Dieses jugendliche zellreiche Gewebe ist es nun, welches in den späteren Stadien von der Synchondrose, wie es in diesem objekt ja schon in den ersten Anfängen hervortritt, beginnend, mehr und mehr in hyalinen Knorpel sich umwandelt.

An der Konvexität der Biegung finden wir an dem achttägigen Präparat einen Einriss in die Zwischenwirbelscheibe, der ebenfalls dicht an der Epiphyse verläuft, tiefer ist als dort und durch zellreiches Gewebe ausgefüllt.

Ein einundzwanzigtägiges Stadium giebt uns vor Allem Veranlassung, die Verhältnisse an der Konkavität noch etwas genauer ins Auge zu fassen. An zwei Gelenken sind Veränderungen eingetreten. An dem einen, sehr stark gebogenen sieht man wieder die Ausfuillung des Winkels durch ein zellreiches Gewebe, welches deutlich von der Unterfläche der Synchondrose ausstrahlt, aber noch keine knorpelige Metamorphose zeigt. Auch an den Diaphysen hat es noch nicht zur Knochenneubildung gefïhrt. An dem schwächer gebogenen Gelenk sind die Processe weiter gediehen. Hier findet sich bereits eine große Menge von Knorpel, der kontinuirlich aus der Zwischenwirbelscheibe hervorgeht und sich unter die eine Diaphyse nur wenig, unter die andere sehr weit, etwa auf ein Viertel ihrer Länge erstreckt und an einer Stelle endet, wo sich bereits ein kleiner Knochenvorsprung gebildet hat. Dazu ist es auf der anderen Seite noch nicht gekommen.

Nach Besprechung der anatomischen Einzelheiten fassen wir nun zunächst die Veränderungen übersichtlich zusammen.

Die mechanischen Folgen der Krümmung sind Annäherung der Knochenränder an der Konkavität und dadurch Kompression des dazwischenliegenden Abschnittes der Intervertebralscheibe, deren innerster Theil, vom Drucke nicht direkt getroffen, sich etwas in den Biegungswinkel hinein vorwölbt. Die Scheibe erhält so im Ganzen eine ausgesprochen kegelförmige Gestalt. In den breiten Abschnitt wird der Chordakern verlagert. Die Epiphysenknorpel und -knochen leiden nur in einzelnen Fällen unter dem Druck und zeigen dann eine mäßige Dickenabnahme; in vielen anderen Fällen waren sie unverändert. Nur ist zu beachten, dass die Epiphysenknochen schon in der Norm an der dorsalen Seite weniger entwickelt sind als an der ventralen. 
An die mechanischen Folgen der Biegung achlieBt sich nun an der Konkavität erstens die Bildung reichlichen hyalinen, ans der Zwischenwirbelscheibe herauswachsenden Knorpels an, zweitens an der angrenzenden Unterfläche der Diaphysen die Entwickelung kegelförmig vorspringender Knochenmassen, die theils periostalen, theils chondrogenen Ursprungs sind. An der Konvexität fanden wir Resorption an den in Folge der Winkelstellung stark vorspringenden Knochenkanten, ferner Neubildung dort, wo es durch die übermäBige Krümmung zu Einrissen in das Gewebe gekommen war.

In welchem Zusammenhang stehen nun alle diese Processe mit der experimentell erzeugten Biegung des Schwanzes? Was veranlasst zunächst die Entstehung des neuen Knorpels und Knochens in dem Krümmungswinkel? So weit ich sehe, kann man hier nicht von einer funktionellen Anpassung in dem Sinne reden, dass die Veränderungen von vorn herein auf Grund der neuen Bedingungen entstünden. Dass sie freilich im ausgebildeten Zustande den durch die Winkelstellung gegebenen Umständen angepasst sind, wird man nicht in Abrede stellen können. Aber ihre Entwickelung hängt davon nicht ab. Wie sollte man auch das Herauswachsen des Knorpels aus der Synchondrose und des Knochens aus der Diaphyse von der veränderten Stellung ableiten können? Zunächst ist ja der sich bildende Knorpel oder besser gesagt, die Unterfläche der Intervertebralscheibe, aus der er sich entwickelt, funktionell ebenso wenig beansprucht wie die angrenzende Unterfläche der Wirbelkörper, auf welche sich Knochen ablagert.

Eine abnorme Beanspruchung findet von Anfang an dort statt, wo die Knochenränder an der Konkavität der Biegung gegen die Zwischenwirbelscheibe angepresst, und dort, wo diese an der Konvexität übermäßig gedehnt wird und durch Zug auf den Knochen einwirkt. Aber es ist in hohem Maße bemerkenswerth, dass diese geänderten statischen Bedingungen Neubildungsvorgänge nur in sehr geringem Maße zur Folge haben. Im Bereich des verstärkten Druckes zeigte sich weder an der Epiphyse, die vielmehr in einzelnen Fällen etwas komprimirt erschien, noch an der Diaphyse eine wesentliche Zunahme der Knochensubstanz, wie man es als Ausdruck einer funktionellen Anpassung vielleicht hätte erwarten sollen. Ebenso wenig war davon an der Konvexität unter der Einwirkung des Zuges die Rede. Allerdings waren die Knochenbälkchen mit Osteoblasten bedeckt, aber da es sich meist um junge Thiere handelte, war das 
um so weniger auffallend, als auch die nicht gebogenen Theile der Schwänze dieselbe Erscheinung darboten. Nur etwas ausgeprägter schien sie dort zu sein und im Zusammenhang damit machte es manchmal den Eindruck, als seien die Knochenbälkchen etwas dicker. Aber nennenswerth waren die Unterschiede niemals und fehlten in manchen Objekten, besonders bei älteren Thieren ganz.

So weit also funktionelle Beanspruchung stattfand, waren die direkten Folgen geringfügig, wo sie aber ganz fehlte, stellten sich ausgesprochene Veränderungen ein, zu deren Verständnis wir meines Erachtens nur auf Grund folgender Überlegungen gelangen. In Folge der Winkelstellung findet an der Konkavität der Krümmung eine Entspannung der Gewebe statt. Die Weichtheile sowohl wie das Periost und die innerste Lage der Intervertebralscheibe nebst Perichondrium verlieren ihre normale Dehnung einmal dadurch, dass sie nicht in einer Linie ansgestreckt sind, sondern einander angenähert werden, und zweitens dadurch, dass sich die Unterfläche der Synchondrose erheblich verringert. Dazu kommt dann drittens, dass sich das längsverlanfende Ligament und die darüber gelegene Muskulatur durch Verkürzung allmählich von der Synchondrose zurïckzieht und dass so zwischen beiden ein größerer Zwischenraum entsteht. Das Alles sind Umstände, welche die normalen gegenseitigen Beziehungen der Gewebsbestandtheile ändern und den Zellen Gelegenheit geben, die ihnen innewohnende Wachsthumsfähigkeit zum Ausdruck zu bringen. Sie vergrößern und vermehren sich so lange, bis die wieder eintretende innere Spannung die weitere Proliferation aufhebt. Damit ist dann in Übereinstimmung, dass die Neubildungsprocesse im Allgemeinen um so hochgradiger sind, je stärker die Winkelstellung ist. Doch muss eine zu weit getriebene Biegung nothwendig wieder eine Zusammendrïckung der im Winkel befindlichen Theile herbeiführen und damit die Wirkung der Entspannung theilweise oder ganz wieder aufheben. Daraus erklärt es sich, dass in dem einundzwanzigtägigen Präparat die Neubildung an der mäßig gebogenen Synchondrose weiter vorgeschritten war als an der Stelle, die so stark geknickt war, dass an der Konvexität ungewöhnlich ausgedehnte Zerreißungen sich eingestellt hatten.

Knorpel- und Knochenbildung halten nun nicht gleichen Schritt. Am raschesten entwickelt sich der hyaline Knorpel und breitet sich oft schon unter der Diaphyse aus, ehe an ihr nennenswerthe osteophytäre Processe auftreten. Je rascher es geschieht, desto weiter 
liegen die ersten Knochenneubildungen, die ja gewöhnlich am Ende der Knorpelwucherung einsetzen, von der Synchondrose entfernt. Je schneller andererseits das Periost sich betheiligt, desto näher rïcken die Osteophyten an die Zwischenwirbelscheibe heran. Manchmal beginnen sie schon genau am Ende des Knochens. Dann führen sie, wie Fig. 2 und 3 sehr deutlich lehren, zu einer Verbreiterung seiner Endfläche. Zwischen die neuen Vorsprünge schiebt sich dann der hyaline Knorpel als eine direkte Fortsetzung der Intervertebralscheibe ein. Aber auch wenn die Knochenneubildung in weiterer Entfernung beginnt, stellen sich schließlich dieselben Verhältnisse ein. Denn der zunächst periostal entstehende Knochen wächst weiterhin auch durch Betheiligung des Knorpels, indem dessen Zellen die bekannte Reihenstellung annehmen (Fig. 3) und indem nun die Markräume des Knochens wie bei der normalen Ossifikation in den Knorpel vordringen. Die Anordnung der Theile bringt es dabei naturgemäß mit sich, dass die neuen Knochenbälkchen senkrecht auf den neuen Knorpel gerichtet, also denen des alten Knochens parallel sind. So ist also die schließliche Folge aller geschilderten Processe eine Verbreiterung der Zwischenwirbelscheibe und der Knochen an der Konkavität der Biegung. Die Struktur des neuen Knochens ist dabei den funktionellen Ansprïichen völlig angepasst, wenn auch die Neubildungsprocesse zunächst ohne den direkten Einfluss der veränderten statischen Inanspruchnahme erfolgten. Späterhin mögen allerdings die Druckverhältnisse in dem Krümmungswinkel nicht ohne Bedeutung für die Anordnung der wachsenden Theile sein.

Die Veränderungen an der Konvexität der Biegung sind leicht verständlich. Die beschriebenen Resorptionserscheinungen lassen sich auf die Wirkung des Druckes zurückführen, welchen die stark gespannten Weichtheile auf die vorspringenden Knochenkanten ausüben. Diese Kompression wirkt ja dauernd ein und ist weit stärker als in der Norm.

Die Neubildungsvorgänge endlich an dem Scheitel der Krümmung sind als regenerative aufzufassen. Sie setzen dort ein, wo mehr oder weniger weitgehende Einrisse in die Intervertebralscheibe oder, wie es gelegentlich der Fall war, auch zwischen Epiphysenknorpel und dem angrenzenden noch wenig festen Diaphysenknochen erfolgten.

Die experimentellen Ergebnisse sind nun gewiss nicht ohne Bedentung für die funktionelle Anpassung des Knochensystems 
uiberhaupt. Freilich wurden sie ja unter anderen Bedingungen gewonnen, als sie beim Menschen vorkommen. Denn in den Versuchen traten die statischen Änderungen plötzlich in voller Intensität auf und blieben in gleicher Stärke bestehen, während sie beim Menschen sich langsam einstellen und erst allmählich verstärken. Also direkt übertragen lassen sich die Versuchsresultate nicht. Aber man wird auch beim Menschen daran denken dürfen, ob nicht bei Verkrïmmungen der Wirbelsäule und Verbiegungen der Gelenke analoge Neubildungsprocesse an der Konkavität und Einschmelzungen an der Konvexität vorkommen und ob nicht darauf die äuPere Gestalt der Knochen wenigstens theilweise zuriekzuführen ist. Ich denke insbesondere an die Keilform der Wirbelkörper und anderer Knochen.

Die Figg. 2 und 3, zum Theil auch 4 und 6 lassen dentlich erkennen, dass die durch Knochenneubildung (Kna und $K n b$ ) erfolgende Verbreiterung der Synchondrosenfläche nicht parallel mit deren ursprünglichen Richtung, also nicht parallel der Epiphysenlinie erfolgt, sondern einen Winkel mit ihr bildet, der je nach der Stärke der Biegung verschieden groß ist. Denken wir uns nun, wir hätten es nicht mit den phalangenähnlichen Wirbelkörpern des Kaninchenschwanzes, sondern den kurzen des Menschen zu thun und stellen wir uns vor, es ginge die Knochenneubildung an der Konkavität eines Wirbels von beiden Synchondrosen aus und betheiligte die ganze Fläche desselben, so müsste er sich einmal verbreitern und zweitens verjüngen, da ja die Knorpelflächen der neuen Knochensubstanz gegen einander geneigt sein würden. Nebmen wir an, es sei in Fig. 2 der Wirbelkörper $b$ etwa quadratisch und durch die punktirte Linie $c$ begrenzt und es habe sich nun bei $d$ ein ähnlicher Knochenvorsprung wie $K n b$ entwickelt, so würde durch die punktirte Linie $e$ die nene Form ungefähr umschrieben sein. Der Wirbel $b$ hätte dann eine annähernde Keilform bekommen.

Nun zeigt allerdings die Synchondrosenfläche des menschlichen Wirbelkörpers eine glatte Beschaffenheit und lässt keinen Winkel erkennen. Aber daran mögen die veränderten Druckverhältnisse einmal in so fern die Schuld tragen, als der Wirbelkörper im Ganzen etwas komprimirt und gegen die Konkavität hin abgeflacht wird. Das trat auch in einzelnen meiner Experimente hervor. So betonte ich bei Besprechung der Fig. 2, dass die Längsachse beider Wirbelkörper mit der Epiphysenlinie einen nach unten offenen spitzen Winkel bildet. Wenn das nicht in allen Versuchen deutlich war, so 
kann man das daraus erklären, dass der Knochen, so weit es sich um ältere Thiere handelte, nicht mehr weich genug war, um einem Druck nachgeben zu können.

Nun wird allerdings jener Winkel an der Synchondrosenfläche durch die Zusammendrückung des Knochens nicht ganz ausgeglichen, aber ob das nicht unter anderen Verhältnissen möglich ist, mag dahingestellt bleiben. Man darf aber zweitens anch daran denken, dass die etwa noch vorspringenden Theile eingeschmolzen werden. Denn ein abnormer Druck macht doch unter allen Umständen so lange Atrophie, bis ein funktionelles Gleichgewicht hergestellt ist.

Auch beim Menschen mag endlich an der Konvexität der gekrümmten Theile eine Einschmelzung vor sich gehen, die den Wirbel hier so weit reducirt, wie er an der Konkavität an Masse gewinnt.

Während aller dieser Umbildungen der äußeren Form werden dann im Inneren der Knochen die Änderungen der Struktur vor sich gehen, für die uns WoLfF so vortreffliche Beispiele beigebracht hat. Da aber die neugebildeten Theile sich den geänderten funktionellen Verhältnissen anpassen, so werden sie mit dem modificirten alten Knochen ein organisches Ganzes bilden.

Ich begnüge mich mit diesen Bemerkungen, die nur darauf hindeuten sollen, in wie weit etwa die experimentellen Ergebnisse auf den Menschen übertragen werden können. Erst fortgesetzte Untersuchungen können uns weitere Aufschlüsse geben. Mir kam es bei meinen Experimenten hauptsächlich darauf an, festzustellen, in welcher Weise abnorme statische Bedingungen den Bau des Knochens modificiren können. Es ergab sich, dass an der abnorm gekrümmten Schwanzwirbelsänle des Kaninchens die alten Knochentheile nur geringe Umbildungen erfuhren, dass vielmehr die Form der Wirbel fast ausschließlich durch Neubildungsvorgänge auf der konkaven Seite der Knochen und der Intervertebralscheibe und durch Resorption an der Konvexität geändert wurde. Die neugebildeten Theile entstanden zunächst ohne eine nachweisbare funktionelle Beanspruchung, nahmen aber schließlich eine den geänderten Bedingungen angepasste Beschaffenheit an. 
Verïndernngen der abnorm gekriimmten Sehwanzwirbelsiule d. Kaninchens. 555

\section{Erklärung der Abbildungen.}

Tafel XXXI.

Die Figuren entsprechen sagittalen Durchschnitten durch eine Zwischenwirbelscheibe und die angrenzenden Theile zweier Wirbelkïrper des Kaninchenschwanzes. Die Wirbelkörper sind mit $a$ und $b$ bezeichnet, $J$ Intervertebralscheibe, $G$ Gallertkern, $E$ Epiphysenknorpel.

Fig. 1. Normaler Schwanz.

Fig. 2. 62 Tage nach Beginn der abnormen Krümmung. $K$ nengebildeter Knorpel. Kna und $K n b$ neugebildeter Knochen. Die Bedeutung der Buchstaben $e, d$ und $e$ ist aus dem Text zu ersehen (pag. 553).

Fig. 3. 42 Tage nach Beginn der Krümmung. $K_{1}$ neugebildeter Knorpel an der Konvexität der Krümmung. $K n$ neuer Knochenkern. Die anderen Bezeichnungen wie vorher.

Fig. 4. 24 tägiger Versuch. Bei $x$ und $z$ finden sich Einschmelzungen des Knochens. Andere Bezeichnungen wie vorher.

Fig. 5. 42 tägiges Präparat. Bezeichnungen wie vorher.

Fig. 6. 72 tägiges Präparat. Bezeichnungen wie vorher.

In den Figuren 2-6, die abnorm gekrümmten Schwanzwirbelsäulen entsprechen, findet sich ïbereinstimmend an der Konkavität der Krümmung Neubildung von Knorpel $(K)$ and Knochen (Kna und $K n b)$. 